\title{
COMANDO ELECTRÓNICO PARA UNA SILLA DE RUEDAS MOTORIZADA
}

Jeremías GARCÍA CABRERA; Oscar G. LOMBARDERO; Víctor J. TORANZOS (*)

\section{RESUMEN}

El presente proyecto nace como una iniciativa de trasferencia tecnológica, del Grupo de Ingeniería en Rehabilitación (GRIER) de la FACENA, cuya finalidad fue el desarrollo de un control electrónico para el comando de una silla de ruedas motorizada, al que se le incorporó el sensado permanente del estado de las baterías y la gestión de la carga de las mismas. Se pretendió obtener un prototipo que responda a los parámetros de los equipos comerciales en cuanto a la simplicidad de manejo, con una interface simple y de considerable autonomía, garantizando la seguridad.

\section{INTRODUCCION}

El presente proyecto está enmarcado en una rama de la ingeniería denominada Ingeniería en Rehabilitación (IR), en la cual se aplican las bases de la ciencia y de la tecnología para desarrollar dispositivos para la rehabilitación o ayuda a personas con algún tipo de discapacidad, ya sea motriz, auditiva o visual. En esta área se realizan perma- nentemente nuevos desarrollos de prótesis $\mathrm{u}$ órtesis en reemplazo de miembros superiores o inferiores para movilidad o brindar una mayor independencia. Todo desarrollo tecnológico tiene como objetivo general proporcionar una solución a un problema mediante el uso de tecnología, y es importante tanto para explicar como para entender un proyecto de este tipo, contextualizar en primera instancia cual es la situación problemática y sus características, para de esa manera tener un punto de partida sobre "por qué" se hace el desarrollo, antes de ver el "cómo" se lo realiza.

\section{Las sillas de ruedas manuales}

Las sillas de ruedas permiten la asistencia al disminuido motriz, en situaciones de parálisis motora de miembros inferiores u otro tipo de discapacidad, como consecuencia de lesiones medulares. La idea que subyace es brindar a la persona la posibilidad de movilizarse de la manera más autónoma posible. Conforme a la discapacidad que presente será el tipo de silla que necesite, dado que, si sólo tiene una parálisis de

(*) GRIER Grupo de Ingeniería en Rehabilitación. FACENA UNNE. 
miembros inferiores, pero posee control de sus miembros superiores, podrá utilizar una silla de ruedas estándar, de movimiento manual, valiéndose de sus brazos para moverla. Por otro lado, si la persona tiene una parálisis severa producida por un traumatismo en la zona cervical, con la imposibilidad de mover libremente los brazos o el torso, las acciones deberían ser realizadas por el movimiento de la cabeza, de los ojos, la presión de aire por la boca, o por comandos de voz. Se deduce que existe una gran diversidad de posibilidades para el control de una silla de ruedas, y que debe adaptarse a cada situación en particular.

A excepción de los aros repulsores con los cuales se ejecuta en forma manual el giro de las ruedas, el resto de las partes son comunes en todos los modelos. Las sillas de propulsión manual pueden ser adaptadas

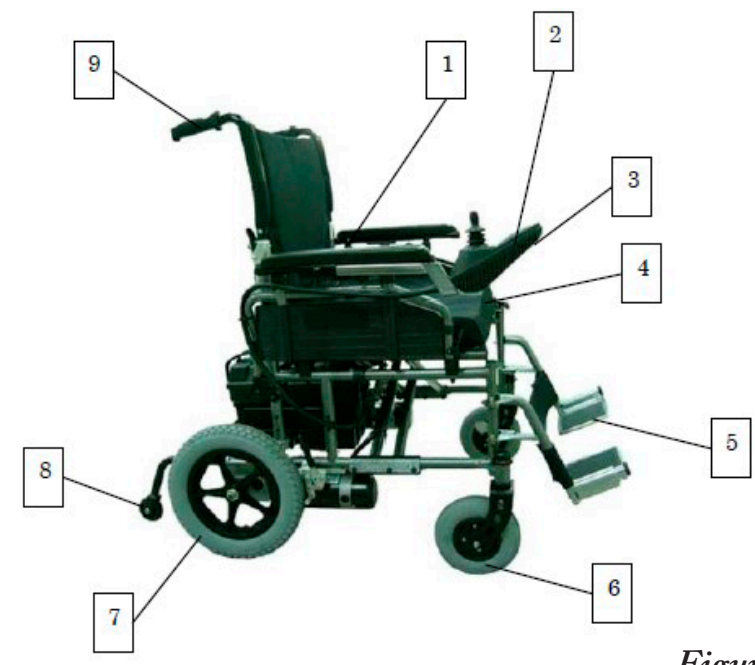

Figura 1

Los parámetros que la diferencia de la anterior silla es la presencia de los motores, las baterías para alimentarlos en forma autónoma, y el control electrónico. Las baterías se encuentran debajo del asiento y con según el tipo de aplicación como ser en el deporte.

\section{Sillas de ruedas motorizadas}

Se caracterizan por efectuar el giro de las ruedas mediante motores eléctricos de corriente continua, los cuales a su vez son comandados por señales provenientes de un joystick, provocando una acción controlada y conjunta del sentido de giro y velocidad. Es más frecuente encontrar personas con cierta movilidad en brazos y manos para comandar la silla. En casos más severos de discapacidad es necesario adoptar otro tipo de comando.

En la Figura 1 se puede observar una silla de ruedas motorizada de la marca "ReActiv." en particular de su modelo "izzyGo-18" con sus partes generales individualizadas:

su centro de masa sobre el eje de las ruedas para evitar desequilibrios. En la Figura 2 se muestra el control de mando, y sus partes más importantes.

Una silla motorizada queda caracteri- 


\section{PANEL DE CONTROL}

1. Botón de encendido/apagado.

2. Indicador de Batería.

3. Joystick.

4. Velocidad máxima / Indicador.

5. Botón para Reducir la Velocidad.

6. Claxon.

7. Botón para Incrementar la Velocidad.

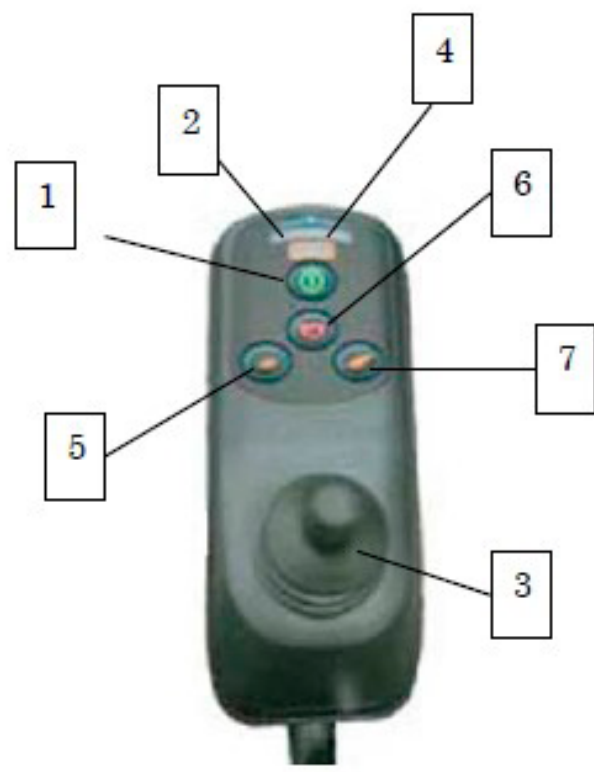

Figura 2

zada con datos como la marca, el modelo, desarrollado cuenta con tres bloques princitipo de baterías, autonomía en km, velocidad pales que pueden apreciarse en la Figura 3. máxima, protecciones, potencia, rodado de Para los ensayos se empleó una silla comerlas ruedas, y pendiente máxima garantizada. cial disponible en el laboratorio.

La idea era reemplazar el control de MATERIALES Y METODOLOGIA fábrica con uno desarrollado íntegramente con componentes fácilmente obtenibles en

El prototipo del control electrónico el mercado local.

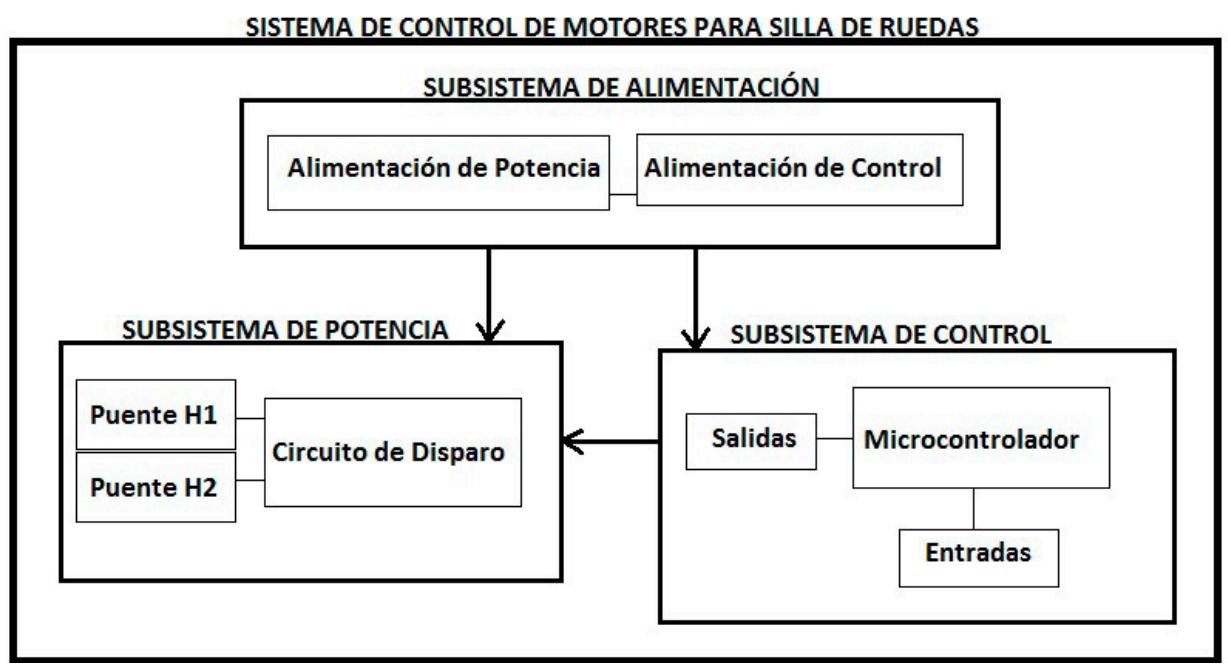

Figura 3 
El Subsistema de Alimentación proporciona dos tensiones aisladas para los otros subsistemas, a partir de las cuales se obtienen las restantes tensiones reguladas necesarias. El Subsistema de Control cuenta con un microcontrolador que toma las señales provenientes del Joystick y genera las señales de salida para el disparo de los puentes $\mathrm{H}$ y las señalizaciones necesarias. El Subsistema de Potencia recibe las señales provenientes del micro y excita los motores de continua, determinando velocidad y sentido de giro.

\section{Subsistema de Control}

Este bloque funcional procesa las señales $\mathrm{X}-\mathrm{Y}$ del joystick, determinando el sentido de giro y la velocidad de ambos motores. También realiza el sensado del estado de las baterías y de la temperatura de los circuitos puente $\mathrm{H}$, que manifestarían eventualmente un consumo excesivo peligroso. Con este fin se ubicaron sensores de temperatura sobre los disipadores de los transistores de potencia. Las seis señales de entrada al micro son

- Eje X del joystick

- Eje Y del joystick

- Sensor de temperatura de Puente 1

- Sensor de temperatura de Puente 2

- Señal de "estado de batería"

- Señal de "cargador conectado"

Por otra parte, a la salida, interactúa principalmente con un circuito combinacional que genera las señales necesarias para accionar el circuito de disparo del subsistema de potencia, una modulación de ancho de pulso, más dos controles de giro por mo- tor (PWM1, PWM2, AD1, AT1, AD2 y AT2). Las señales de salida son

- Control de motores (6)

- Pantalla LCD (7)

- Pin de apagado de la pantalla LCD

- Pin de control de luz del LCD

- LED de Alta Temperatura

- LED de Baja Tensión

- Alarma sonora para alerta al usuario

- Relé de conexión/desconexión

\section{Microcontrolador:}

El CI utilizado para este proyecto es el PIC16F877A de la empresa Microchip, el mismo consta de 40 pines, entre ellos las E/S suficientes para los que exige la aplicación, como así también cuenta con dos módulos de generación de PWM, siete entradas de conversión $\mathrm{A} / \mathrm{D}$, tres temporizadores, $8 \mathrm{k}$ de memoria Flash de programa. La distribución de $\mathrm{E} / \mathrm{S}$ de los pines del microcontrolador se realizó de la siguiente forma:

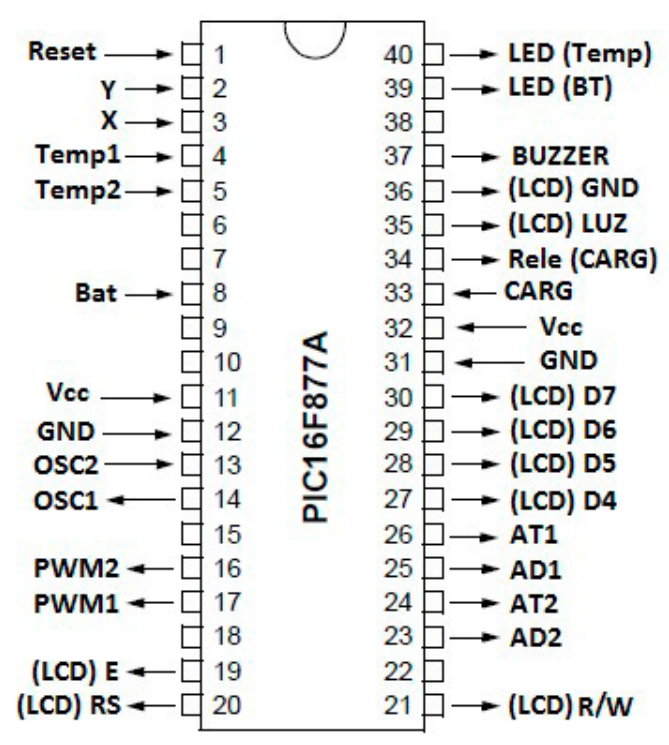

Figura 4 
Las salidas (digitales en su totalidad con valores de $0 \mathrm{v}$ para un Cero lógico y $5 \mathrm{~V}$ para un Uno lógico) corresponden a:

- $\quad$ PWMx = señal modulada en ancho de pulso para el Motor $\mathrm{x}$.

- $\quad \mathrm{ATx}$ = señal para indicar que el $\mathrm{Mo}^{-}$ torX debe girar hacia "ATRAS".

- $\mathrm{ADx}=$ señal para indicar que el MotorX debe girar hacia "Adelante".

- (LCD) Dx = señal de datos de la pantalla

- (LCD) E = señal de "Habilitación"

- (LCD) RS = señal de "Selección de Registros" del LCD.

- (LCD) $\mathrm{R} / \mathrm{W}$ = señal de "Lectura/ Escritura" del LCD.

- (LCD) LUZ = señal que enciende o apaga la luz de la pantalla.

- (LCD) GND = señal que conecta o deja flotante la masa del LCD.

- $\quad$ BUZZER = señal que activa la alarma sonora.

- $\operatorname{LED}($ Temp) = señal que activa el led de Error por Alta Temperatura.

- $\operatorname{LED}(\mathrm{BT})$ = señal que activa el led de Error por Baja Tensión.

- Relé $(\mathrm{CARG})$ = señal que activa el relé que conecta el cargador de baterías.

Por otra parte, tenemos

- $X$ e $Y=$ señales analógicas que varían de 0 a $5 \mathrm{~V}$, con un valor en estado de reposo del joystick de 2,5 +/- 0,1V para ambos casos.

- $\quad$ TempX = señal proveniente de circuitos con $\mathrm{AO}$ que acondicionan la tensión proveniente de los sensores de temperatura tipo LM35, cuyo valor oscila entre los 2 y
$3 \mathrm{~V}$.

- $\quad$ Bat = señal del estado de la batería, proveniente de un divisor resistivo ajustable de relación 1:6, para los valores críticos de la batería varía ente 3,9 y 4,4V.

- $\mathrm{CARG}=$ señal digital que notifica al microcontrolador de si el Cargador de Batería está o no conectado.

El programa que se implementó gestiona

- La interfaz gráfica mediante el display LCD.

- El disparo de alarmas de apagado.

- La carga de la batería.

- El movimiento de la silla mediante el cálculo de los PWM enviados a cada motor en función de la posición relativa del joystick.

La forma en la que el microcontrolador calcula los ciclos de trabajo de los PWM y los sentidos de giro se basa en la división del espacio del joystick en distintas zonas de trabajo con un cálculo asociado a cada uno. Este proceso se presenta en la Figura 5.

Las variables duty1 y duty2 son los ciclos de trabajo con lo que se actúa sobre cada motor y los valores $\mathrm{X}$ a $\mathrm{Y}$ son el resultado de las conversiones $\mathrm{A} / \mathrm{D}$ de cada eje del joystick.

Conforme el joystick varíe su posición por la región de trabajo, el sistema calcula tanto los ciclos de trabajo que definen la velocidad, como también el código que fija el sentido de giro. Si graficamos las velocidades de ambos motores en función de la posición del joystick girando en sentido anti-horario, suponiendo una inclinación 


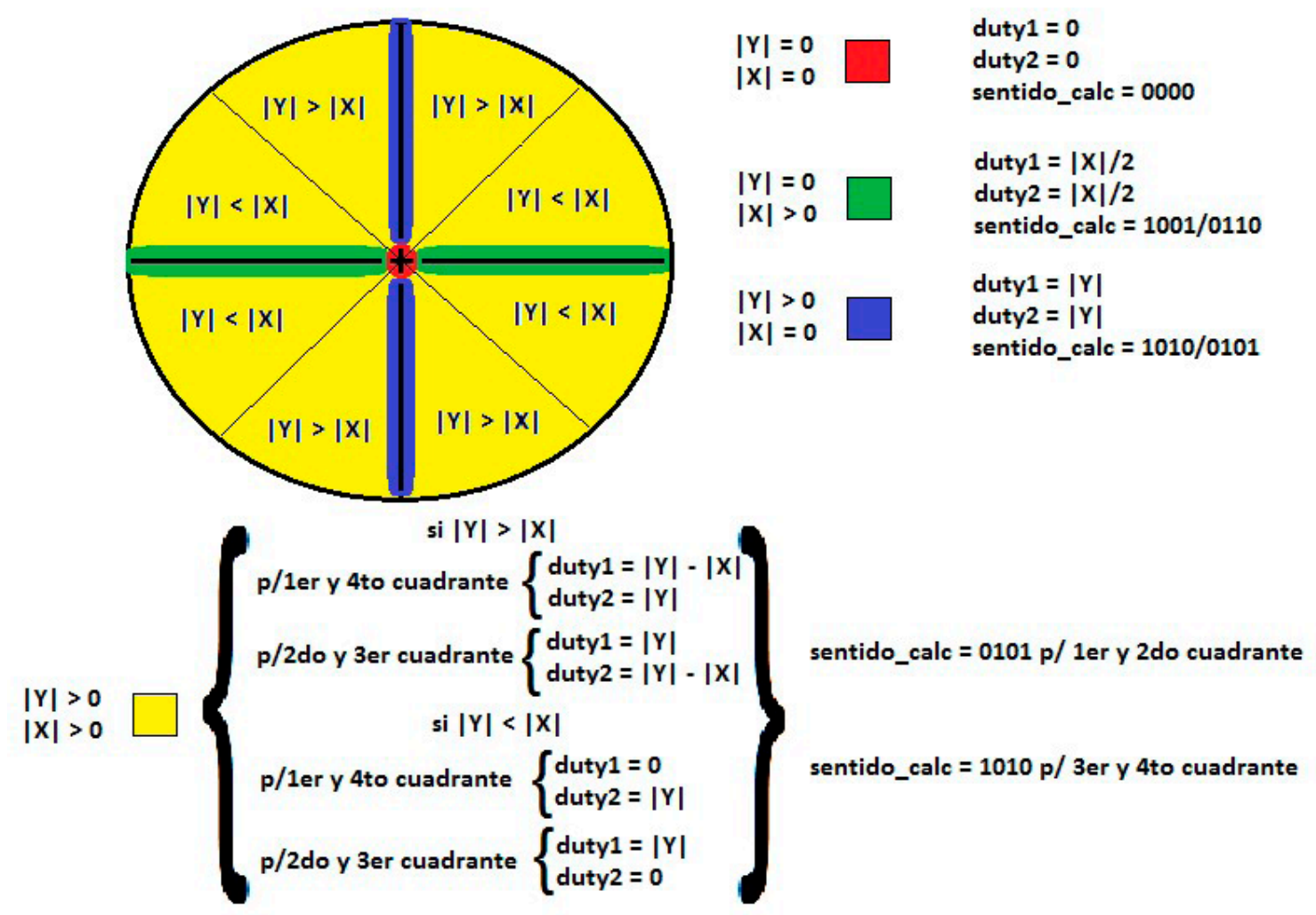

Figura 5

máxima, podemos inferir el movimiento la Figura 6 se representa el nomograma de de la silla según la posición del mando. En cálculo.

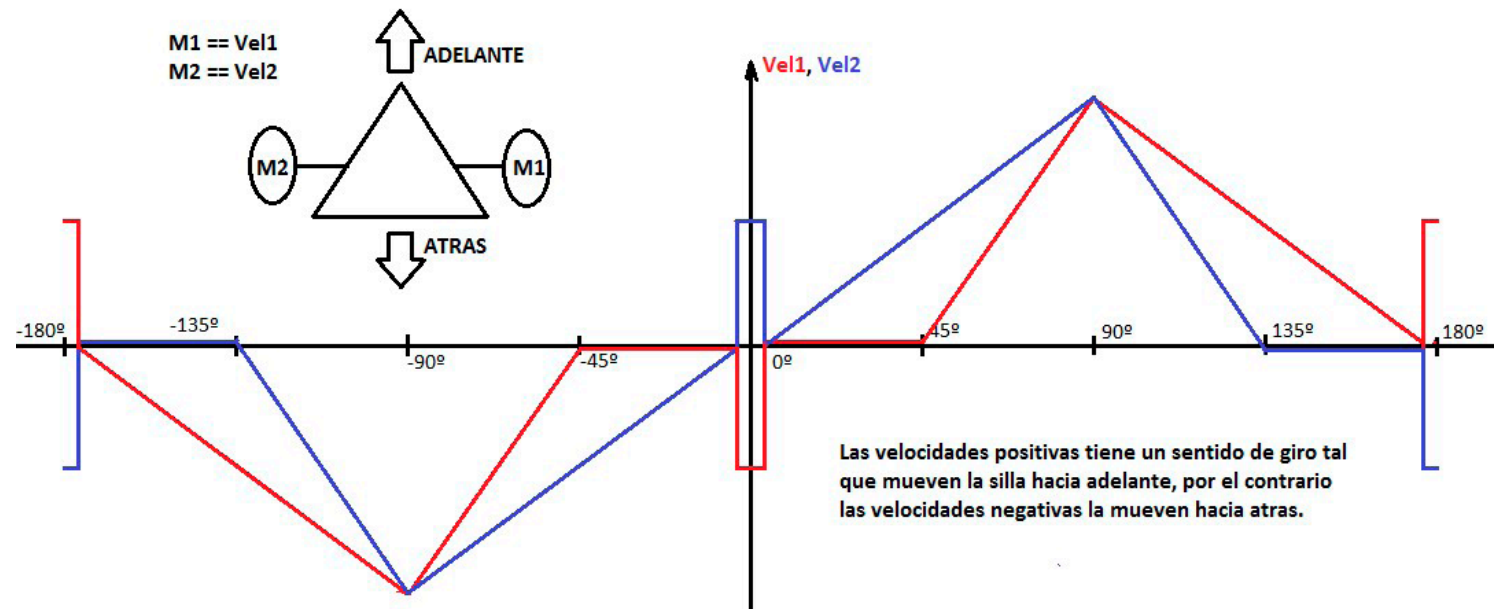

Figura 6 


\section{Subsistema de Potencia}

Este módulo recibe las señales del microprocesador en formato PWM para excitar el disparo de los transistores de potencia por los cuales circulara la corriente de los motores, que están conectados a la fuente de poder principal conformada por las dos baterías en serie.

El Circuito de Disparo tiene dos configuraciones distintas que se repiten en cada puente. Esta diferencia es necesaria por la posición relativa de los transistores que pueden encontrarse en la parte superior del puente $\mathrm{H}$ (drenador $\mathrm{a}+\mathrm{B}$ ) o bien en la parte inferior (surtidor a tierra). Dependiendo a que tensión este referenciado el drenador del MOSFET, se determina qué nivel de tensión es necesario para superar el umbral de disparo VGS (tensión entre compuerta y surtidor), que según la hoja de datos tiene un valor máximo de VGS $=4 \mathrm{~V}$. Los transistores de la parte superior del puente al momento de ser disparados, tendrán sus surtidores referenciados al borne positivo de la carga, lo que hace que tenga una referencia igual a la fuente de alimentación (alrededor de $25 \mathrm{~V}$ ) valor que sumado a VGS es que mínimo que deberá aplicarse a la compuerta de los transistores para poder dispararlos. Por su parte los MOSFET de la parte inferior del puente al tener el surtidor a los $0 \mathrm{~V}$ de la masa de la fuente, la VGS necesaria es la del umbral natural del transistor. Los esquemáticos del circuito de disparo para los transistores superiores (Fig. 9) e inferiores (Fig. 8) de cada puente son los siguientes:

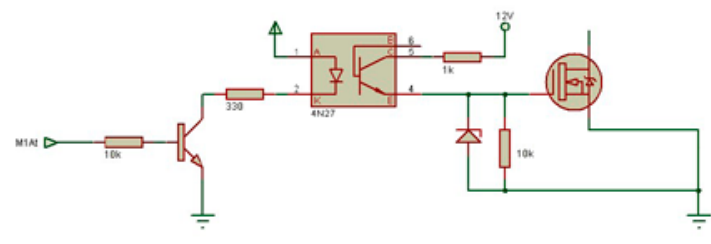

Figura 7

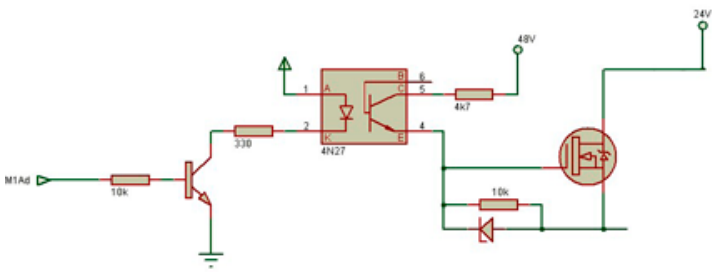

Figura 8

Para completar el circuito de disparo se implementó un doblador de tensión.

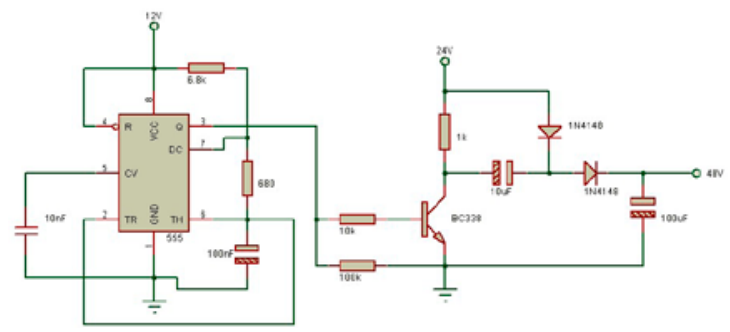

Figura 9

Si bien la salida de este tipo de circuitos no está preparada para entregar mucha corriente, no hay inconveniente ya que se utiliza para disparar dos MOSFET a la vez como máximo, y las corrientes que demandan estos disparos están limitadas por las resistencias del colector de los fototransistores, por lo que el valor máximo que se le exige a este circuito es de $6,4 \mathrm{~mA}(15 \mathrm{~V} /$ $4700 \mathrm{Ohm}=6,39 \mathrm{~mA}$ ), valor que entrega el doblador de tensión sin comprometer significativamente la tensión de salida.

La segunda etapa del subsistema de potencia son los puentes $\mathrm{H}$ que interac- 
túan en forma directa con los motores. Ambos son circuitos idénticos y tienen la disposición de la Figura 10.

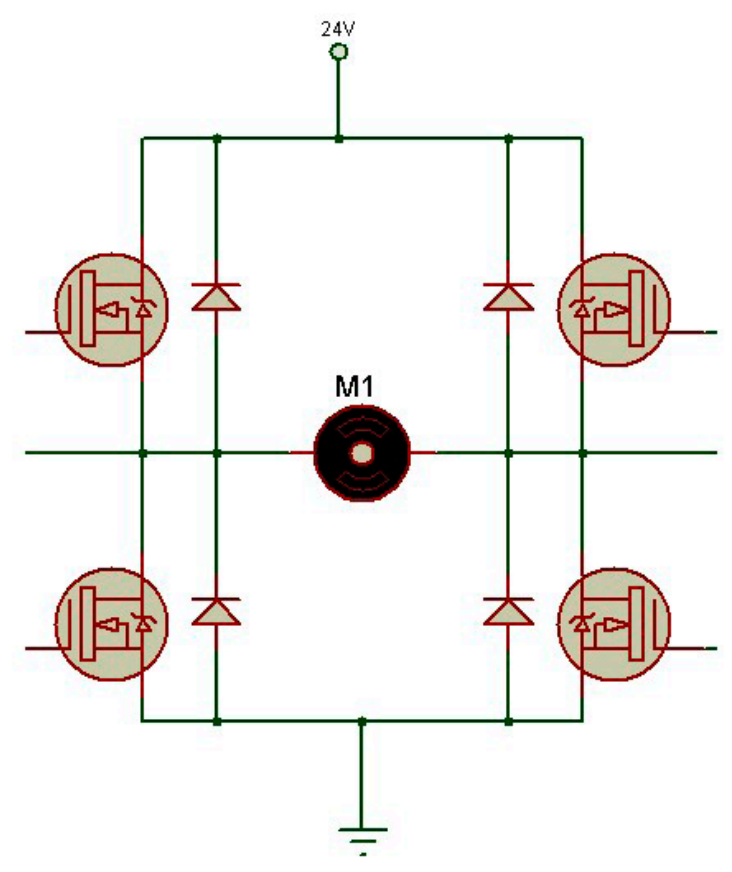

Figura 10

Cada par de transistores con posiciones cruzadas (superior derecho con inferior izquierdo y viceversa) recibe la misma señal de PWM como señal de disparo, correspondiendo a un sentido de giro en particular. Por programa se garantiza que en ningún momento se saturen los transistores de una misma rama, porque ocasionaría un cortocircuito en el puente y la destrucción de los componentes.

\section{Subsistema de Alimentación}

Cuenta con dos sistemas de alimentación simples de masa común, lo cual permite tomar desde el microcontrolador una muestra de la tensión de la batería con sólo un divisor resistivo, y por otro lado permite que los bornes positivos de ambas fuentes estén totalmente desvinculados.

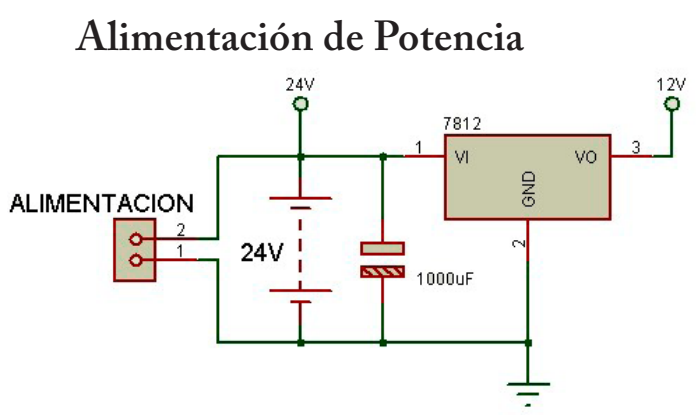

Figura 11

Los bornes de alimentación están conectados directamente al arreglo en serie de las baterías, con lo cual se obtiene una fuente de CC de $25 \mathrm{~V}$, dependiente del estado de carga. En paralelo se colocó un capacitor electrolítico como filtro y por último un regulador LM7812 que proporciona la tensión para el circuito de disparo, para excitar los MOSFET inferiores de cada puente y alimentar el LM555 del doblador de tensión. Los bornes de las baterías van directamente a los extremos de los puentes tipo $\mathrm{H}$.

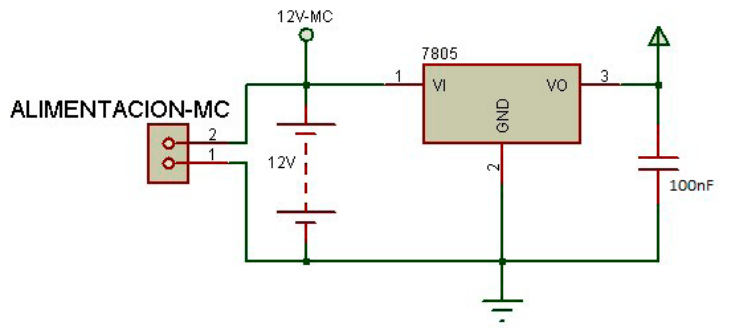

Figura 12

\section{Alimentación de Control}

En este caso, los bornes se conectan a una fuente de $12 \mathrm{~V}$ distinta de las baterías 
y de baja potencia, ya que excita la bobina del relé y un regulador LM7805 para alimentar todo el subsistema de control, de bajo consumo. A la salida del regulador se colocó un capacitor cerámico de $100 \mathrm{nF}$ para filtrar la alimentación del micro de posibles interferencias.

El circuito impreso fue desarrollado en una placa doble faz de fibra de vidrio, con una medida de $20 \times 15 \mathrm{~cm}$. En la Figura 13 y 14 se presenta la vista en 3D del circuito impreso con los componentes.

\section{CONCLUSION}

El desarrollo de este proyecto tuvo

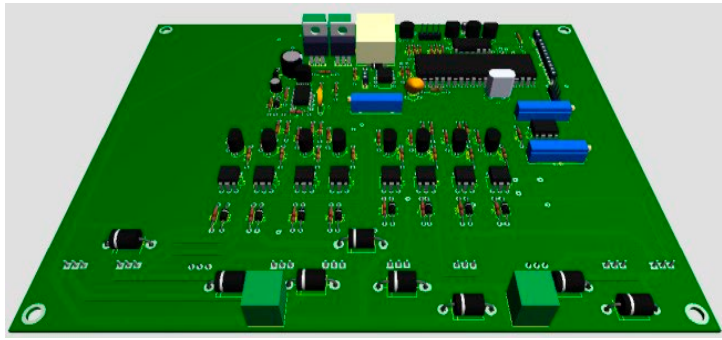

Figura 13 como objetivo principal el diseño y construcción de un prototipo basado en un producto que, si bien está comercialmente disponible, tiene una alto costo, lo que dificulta el acceso a un sector de la población de bajos recursos. Considerando que además son personas con discapacidad, se avanzó con la construcción de este prototipo, que cumplió con las expectativas iniciales de comandar electrónicamente una silla de ruedas motorizada, con un consumo moderado, autonomía equivalente de los productos disponibles comercialmente y una interfaz gráfica con el usuario sencilla.

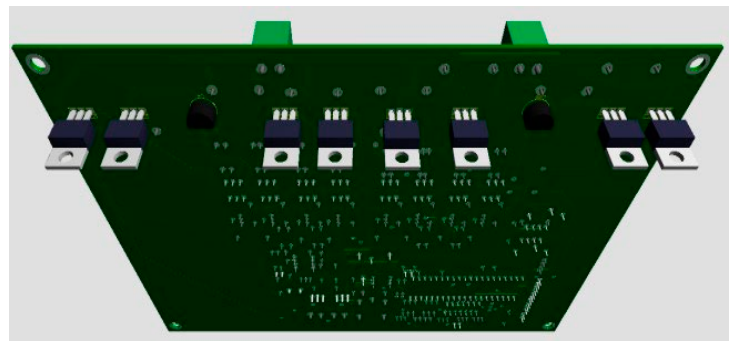

Figura 14

\section{BIBLIOGRAFÍA}

[1] Tortora y Derrickson, "Introducción al Cuerpo Humano: Fundamentos de Anatomía y Fisiología.", séptima edición, 2007.

[2] Timothy Maloney, "Electrónica Industrial Moderna”, quinta edición, 2006.

[3] Saravia y Coria, "Arquitectura y programación de microcontroladores PIC.”, primera edición, 2010.

[4] Usategui, Yesa y Martinez, "MICROCONTROLADORES PIC Diseño práctico de aplicaciones SEGUNDA PARTE: PIC16F87X, PIC18FXXXX.”, segunda edición, 2006.

[5] Microchip Technology Inc., "PICmicro ${ }^{\mathrm{TM}}$ MidRange MCU Family Reference Manual”, 1997.

[6] Custom Computer Services Inc., "C Compiler
Reference Manual”, cuarta edición, 2009.

[7] Hugo Cantore, "Causas y consecuencias de la discapacidad.", asignatura Ingeniería en Rehabilitación, Universidad Nacional de Córdoba, 2010.

[8] Hugo Cantore, “Deportes y discapacidad.”, asignatura Ingeniería en Rehabilitación, Universidad Nacional de Córdoba, 2010.

[9] Celestino B. Brutti, Aníbal J. Sattler, Darío Albacetti, Alberto R. Canavelli y Carlos B. Donisi, "Motorización de Sillas de Ruedas Convencionales: Unidad totalmente desarmable y con exclusivo sistema de desacople de motores para tracción manual", Facultad de Ciencias de la Vida y la Salud - Universidad Autónoma de Entre Ríos 